Math. Nachr. 226 (2001), $153-176$

\title{
Newton's Problem of the Body of Minimal Resistance in the Class of Convex Developable Functions
}

By T. Lachand - Robert of Paris and M. A. Peletier of Amsterdam

(Received October 29, 1999; revised version February 1, 2000; accepted March 31, 2000)

Abstract. We investigate the minimization of Newton's functional for the problem of the body of minimal resistance with maximal height $M>0$ [4] in the class of convex developable functions defined in a disc. This class is a natural candidate to find a (non-radial) minimizer in accordance with the results of [9].

We prove that the minimizer in this class has a minimal set in the form of a regular polygon with $n$ sides centered in the disc, and numerical experiments indicate that the natural number $n \geq 2$ is a non-decreasing function of $M$. The corresponding functions all achieve a lower value of the functional than the optimal radially symmetric function with the same height $M$.

\section{Introduction}

There has been a recent revival of interest in Newton's problem of the body of minimal resistance. In modern terms this problem can be formulated as the minimization problem

$$
\inf _{U \in \mathcal{C}} \mathcal{F}(U), \quad \text { with } \quad \mathcal{F}(U)=\int_{\Omega} \frac{d x}{1+|\nabla U|^{2}}
$$

Here $\Omega$ is a smooth subset of $\mathbb{R}^{2}$; the graph of the function $U$ represents the form of a three-dimensional body, and the functional $\mathcal{F}$ models the resistance experienced by this body as it moves through a cloud of gas particles. We refer to [4] for a detailed discussion of the model and the history of this problem.

The form of $\mathcal{F}$ favours functions $U$ with rapid oscillations. Because of this fact, the choice of the class $\mathcal{C}$ of admissible functions is a delicate issue. A number of different choices have been explored in the literature $[4,5,6,7,2]$, but the most interesting

1991 Mathematics Subject Classification. Primary: 49K99; Secondary: 49K30.

Keywords and phrases. Body of minimal resistance, convexity constraint, non-convex minimization, developable functions. 
one, from a mathematical viewpoint, seems to be

$$
\mathcal{C}=\{U: \Omega \rightarrow[0, M]: U \text { is convex }\}
$$

We shall adopt this definition of $\mathcal{C}$ throughout the further discussion. The scalar constant $M>0$ is a parameter whose role will become clear below.

When $\Omega$ is a ball in $\mathbb{R}^{2}$ — which we shall assume from now on - the function $\mathcal{F}$ and the set $\mathcal{C}$ are rotationally invariant. Newton exhibited a function $U$ [8] that is radially symmetric and minimizes the functional $\mathcal{F}$ among all radially symmetric members of $\mathcal{C}$. This function is smooth and strictly convex, except on a circular set where its derivative jumps (see Figure 1, the rightmost shape). For a long time it was implicitly assumed that the minimizer of $\mathcal{F}$ among the whole of $\mathcal{C}$ is necessarily radially symmetric, and therefore coincides with the function found by NEWTON. However, the symmetry of the general minimizer was never proved, and in 1996 the converse was demonstrated by Brock, Ferone, and KaWohl [3]: when $\Omega$ is a ball, the minimizer of $\mathcal{F}$ over $\mathcal{C}$ is necessarily non-radially symmetric. The proof consisted of remarking that the second derivative of $\mathcal{F}$, calculated at Newton's function, had a negative direction that was admissible. Therefore the functional was not minimal.

This result naturally opened the hunt on the true form of the minimizer. In [9] we proved a first step in this direction:

Theorem 1.1. Let $U$ achieve (1.1), and let $\omega \subset \Omega$ be an open set. Then $U$ is not strictly convex on $\omega$.

The class of non - strictly convex functions on $\Omega$ is still relatively large. In this paper we jump the gun, and investigate the functional $\mathcal{F}$ on a much smaller set of functions $\mathcal{C}_{d}$. The set $\mathcal{C}_{d}$ is defined to contain all functions $U \in \mathcal{C}$ such that the graph of $U$ is the convex envelope in $\mathbb{R}^{3}$ of the sets $\partial \Omega \times\{M\}$ and $N_{0} \times\{0\}$, where

$$
N_{0}=\{x \in \Omega: U(x)=0\} .
$$

Alternatively (and equivalently): $U$ belongs to $\mathcal{C}_{d}$ whenever $U$ is convex, takes values between 0 and $M$, satisfies $U(\partial \Omega)=M$, and has no extremal points in $\Omega \backslash \overline{N_{0}}$. Figure 2 shows the contour lines of a typical function $U$. Note that for elements of $\mathcal{C}_{d}$ the convex set $N_{0}$ completely characterizes the function.

The reason for considering this set of admissible functions is the following conjecture:

Conjecture 1.2. Let $U$ solve (1.1). Then $U \in \mathcal{C}_{d}$.

As supporting evidence for this conjecture we mention of course Theorem 1.1. Indeed, if we know that $U$ is of class $C^{2}$ in $\Omega \backslash \overline{N_{0}}$, then the conjecture follows from a well-known geometrical property: any regular convex surface with zero Gaussian curvature is indeed a so-called developable surface, and can be extended up to a singular line called line of striction. Unfortunately, no similar result seems to be known for convex surfaces without any a priori additional regularity; moreover, if $\nabla U$ has discontinuities in $\Omega \backslash \overline{N_{0}}$, then $U$ is only a piecewise developable function. This is why the conjecture is still an open question. 
In this paper we thus investigate the problem

$$
\inf _{U \in \mathcal{C}_{d}} \mathcal{F}(U)
$$

We obtain the following result:

Theorem 1.3. Let $M>0$ be given. If $U$ solves (1.2), then the set $N_{0}$ is a regular polygon centered in $\Omega$.

This theorem is proved in two steps. In Section 4 we show that $N_{0}$ is necessarily a polygon. In Section 5 we show in addition that this polygon must be regular and centered.

We have computed the value of the functional $F$ for the different regular polygons ( $m=2,3,4$, etc.) with an explicit formula (see Appendix A). It turns out, from numerical experiments, that there exists a decreasing sequence $\left(M_{n}\right) \subset \mathbb{N}, n=2,3, \ldots$, with $M_{2}=\infty$, with the following property: If $M_{n+1}<M<M_{n}$, and if $U$ solves (1.2), then the set $N_{0}$ is a regular polygon with $n$ sides.

This can be seen in Figure 1, where the analytic curves formed by the value of $F$ for $n$-sided regular polygons are plotted; the optimal curve is a piecewise combination of these. We have also shown in this graph the value of the functional for the radial minimizer given by NEWTON.

The critical values $M_{n}$ are given in Table 1 .

Table 1: Critical values of $M$ for regular polygons.

$$
\left|\begin{array}{ll}
M_{3} & 1.179535875 \\
M_{4} & 0.754344515 \\
M_{5} & 0.561232469 \\
M_{6} & 0.447571675 \\
M_{7} & 0.372163842 \\
M_{8} & 0.318383452 \\
M_{9} & 0.278081912
\end{array}\right|
$$

\section{The problem in $\mathcal{C}_{d}$}

The members of $\mathcal{C}_{d}$ are completely characterized by their set $N_{0}$, and using the convexity of this set we introduce a more convenient representation. Consider the function $U$ of which the contour lines are drawn in Figure 2.

We assume that $\Omega$ is the unit ball, and we denote by $s$ the arclength coordinate along $\partial \Omega=S^{1}$, in the positive direction; $x(s)$ is the point at $s$, and let $\ell(s)$ be the tangent of $N_{0}$ parallel to $x^{\prime}(s)$, as shown in the figure. We define $u(s)$ to be the signed distance of $\ell(s)$ to the origin, i. e. $u(s)=d(\ell(s), 0)$ if $\ell$ separates 0 from $x(s)$, and $u(s)=-d(\ell(s), 0)$ otherwise. Then $u(\cdot)$ completely characterizes $N_{0}$, and to every convex set $N_{0} \subset \Omega$ there corresponds a function $u: S^{1} \rightarrow[-1,1]$. 


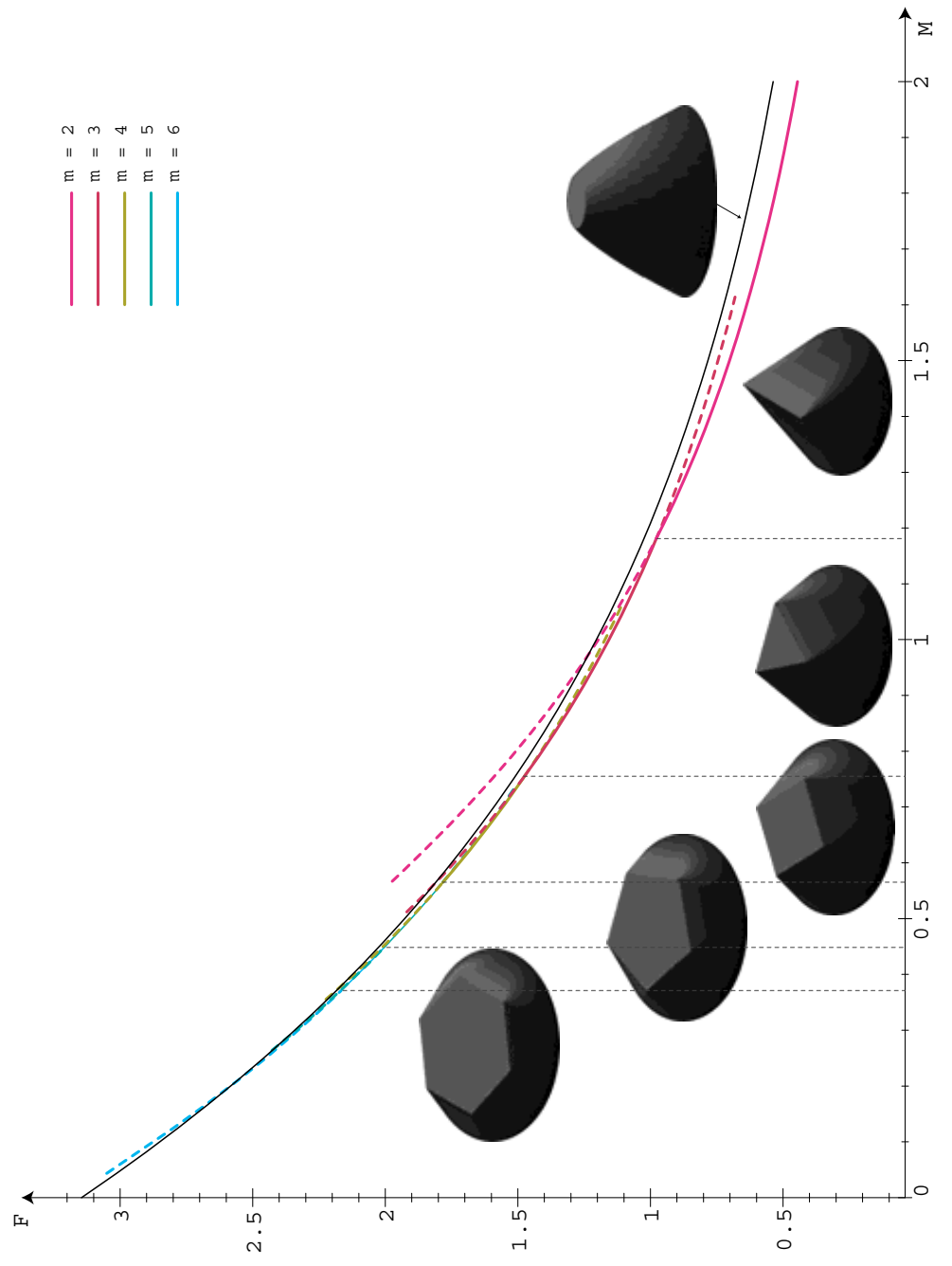

Figure 1: Value of the functional $F$ for optimal regular functions; the thinner line up shows the value for Newton's radial minimizer. We plot the graph of $U$ inverted, as a concave function, since the human eye is accustomed to viewing objects from above. 
We now express the convexity condition and the functional $\mathcal{F}$ in terms of $u$. If $\ell(s)$ intersects $N_{0}$ in one point, then we define $y(s)$ to be this intersection point. If $\ell(s)$ has multiple intersections with $N_{0}$, then $y(s)$ is not well-defined, and the function $y$ is discontinuous at such a point. Denoting the radial and tangent unit vectors at $x(s)$ by $e_{r}(s)$ and $e_{s}(s)$, we have

$$
u(s)=y(s) \cdot e_{r}(s) .
$$

Hence $u^{\prime}(s)=y(s) \cdot e_{s}(s)$ since $y^{\prime}(s) \cdot e_{r}(s)=0 ;$ and

$$
u^{\prime \prime}(s)=y^{\prime}(s) \cdot e_{s}(s)-y(s) \cdot e_{r}(s)=\left|y^{\prime}(s)\right|-u(s)
$$

implying

$$
u+u^{\prime \prime}(s)=\left|y^{\prime}(s)\right| \geq 0 .
$$

This is an important result: the condition $u+u^{\prime \prime} \geq 0$ (in the sense of distributions on $S^{1}$ ) is equivalent to the statement that the corresponding set $N_{0}$ is convex. In the following we shall always assume that $u$ satisfies this condition.

While discussing this point, note that since $u+u^{\prime \prime}=\left|y^{\prime}(s)\right|$, the distribution $u+u^{\prime \prime}$ is in fact a Radon measure. The support of the singular part of this measure coincides with the values of $s$ at which $y$ is not continuous, or equivalently, at which $\ell(s)$ intersects $N_{0}$ in a line segment of non-zero length. The value of the singular part of $u+u^{\prime \prime}$ at such a point is equal to the jump in $y$, which in turn equals the length of the line segment. At all other points $s$ the singular part of $u+u^{\prime \prime}$ is zero, and $y$ is continuous.

On the straight line segment $[x, y]$ the gradient of $U$ is constant, and its length equals $M /\left((x-y) \cdot e_{r}\right)=M /(1-u)$. Hence, using the notation $f(t)=1 /\left(1+|t|^{2}\right)$, we find

$$
\begin{aligned}
\mathcal{F}(U) & =\int_{\Omega} f(\nabla U) d x \\
& =\frac{1}{2} \int_{S^{1}} f(0) y(s) \wedge y^{\prime}(s) d s+\frac{1}{2} \int_{S^{1}} f\left(\frac{M}{1-u(s)}\right)(x-y) \wedge\left(x^{\prime}+y^{\prime}\right)(s) d s \\
& =\frac{1}{2} f(0) \int_{S^{1}} u\left(u+u^{\prime \prime}\right)+\frac{1}{2} \int_{S^{1}} f\left(\frac{M}{1-u}\right)(1-u)\left(1+u+u^{\prime \prime}\right) .
\end{aligned}
$$

Hence since $f(0)=1$, we have to minimize:

$$
F(u)=\frac{1}{2} \int_{S^{1}} u\left(u+u^{\prime \prime}\right)+\frac{M}{2} \int_{S^{1}} \Phi\left(\frac{1-u}{M}\right)\left(1+u+u^{\prime \prime}\right)
$$

where

$$
\Phi(x):=x f(1 / x)=\frac{x^{3}}{1+x^{2}}
$$

in the set $X(-1,1)$, where we define

$$
X(a, b):=\left\{u \in W^{1, \infty}\left(S^{1}\right) ; a \leq u \leq b, u+u^{\prime \prime} \geq 0\right\} .
$$

Note that (2.1) is meaningful, as integrals of a product of a continuous function and a Radon measure. 


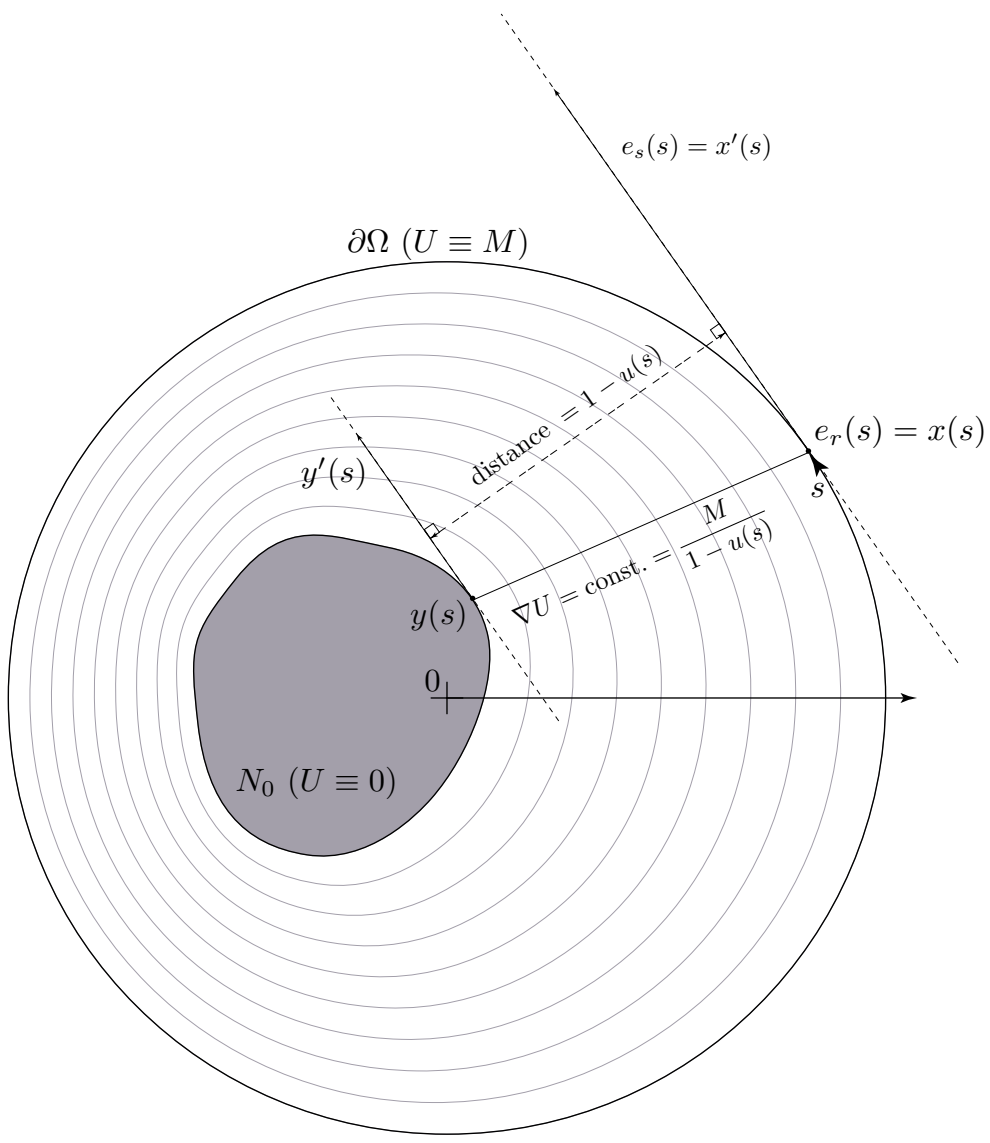

Figure 2: Parametrization of $N_{0}$.

It is sometimes convenient to write $F$ in the form:

$$
2 F(u)=\int \Psi(u)\left(1+u+u^{\prime \prime}\right)-\int u
$$

where

$$
\Psi(t)=t+M \Phi\left(\frac{1-t}{M}\right)=\frac{t M^{2}+(1-t)^{2}}{M^{2}+(1-t)^{2}} .
$$

Integrating by parts, this can also be written in the form:

$$
F(u)=\frac{1}{2} \int_{S^{1}} \eta(u)-u^{\prime 2} \Psi^{\prime}(u)
$$

where 


$$
\begin{aligned}
\eta(t) & :=-t+(1+t) \Psi(t)=\frac{M^{2} t^{2}+(1-t)^{2}}{M^{2}+(1-t)^{2}} \\
\Psi^{\prime}(t) & =M^{2} \frac{M^{2}-(1-t)^{2}}{\left(M^{2}+(1-t)^{2}\right)^{2}} .
\end{aligned}
$$

We shall also use

$$
\Psi_{1}(t):=\Psi(t)-1
$$

\section{An estimate of $\nabla U$}

In [4] it was proved that if $U$ achieves (1.1), then $|\nabla U| \notin(0,1)$. This fact is related to the concaveness of $f(t)=1 /\left(1+|t|^{2}\right)$ near zero. The radially symmetric minimizer $U_{N}$ presented by Newton satisfies this condition, and in fact achieves the limit case: close to $N_{0},|\nabla U|$ approaches the value 1 .

The bound on $u$ of Lemma 3.1 below is a reformulation of this result: since $|\nabla U|=$ $M /(1-u)$, the inequality $|\nabla U| \geq 1$ is equivalent to

$$
u \geq 1-M .
$$

If Conjecture 1.2 were proved, then (3.1) would follow directly from [4]. Since we have no proof for the conjecture, we state here an independent proof.

Lemma 3.1. Let $u$ solve problem (4.1). Then $u \geq 1-M$.

Proof. To force a contradiction we assume that $u\left(s_{0}\right)<1-M$, and without loss of generality we also assume that $s_{0}$ is a local minimum of $u$. There are two possibilities: either $u^{\prime}$ jumps at $s=s_{0}$, or $u^{\prime}$ is continuous. We first consider the former case.

Note that $u<1-M$ is equivalent to $\Psi^{\prime}(u)<0$. For small $\eta>0$, to be chosen later, we define $\tilde{u}$ to be continuous on $S^{1}$, and to satisfy in addition

$$
\tilde{u} \equiv u \text { on } S^{1} \backslash\left(s_{0}-\eta, s_{0}+\eta\right) \quad \text { and } \quad \tilde{u}+\tilde{u}^{\prime \prime}=0 \text { on }\left(s_{0}-\eta, s_{0}+\eta\right) .
$$

The geometrical interpretation of $\tilde{u}$ is that of a polygon similar to that of $u$, but where the side corresponding to $s_{0}$ is split into two sides, which are then slightly dented outwards.

We set $v=\tilde{u}-u$, and require $\eta$ to be small enough to ensure that $\Psi^{\prime}(u)<0$ on the support of $v$. On the basis of (2.4) the derivative of $\varepsilon \mapsto 2 F(u+\varepsilon v)$ at $\varepsilon=0$ is given by

$$
2 F^{\prime}(u) \cdot v=\int \Psi(u) v^{\prime \prime}+\int \Psi^{\prime}(u) v\left(1+u+u^{\prime \prime}\right)+\int(\Psi(u)-1) v .
$$

Note that since $v \geq 0$ and $\Psi(\cdot)-1 \leq 0$, the last two terms are negative; in order to obtain a contradiction we only need to show that the same is true for the first.

The function $v^{\prime \prime}$ is a Radon measure with zero integral, i. e. $\int v^{\prime \prime}=0$. The positive part $\left(v^{\prime \prime}\right)_{+}$is concentrated at $s=s_{0} \pm \eta$. Noting the sign of $\Psi^{\prime}$ we have $\Psi(u(s)) \geq$ $\Psi\left(u\left(s_{0}+\eta\right)\right)$ for $s_{0}-\eta \leq s \leq s_{0}+\eta$. Therefore

$$
\int \Psi(u) v^{\prime \prime}=\int\left[\Psi(u)-\Psi\left(u\left(s_{0}+\eta\right)\right)\right] v^{\prime \prime}<0 .
$$


This proves the Lemma for the first case.

We next turn to the second possibility, and assume that $u^{\prime}$ is continuous at $s=s_{0}$. We choose $\eta>0$ and define $v(s)=\left[u\left(s_{0}+\eta\right)-u(s)\right]_{+}$. Repeating the argument, we again have $v \geq 0$, so that the last two terms in (3.2) are negative. By a similar reasoning it follows that

$$
\int \Psi(u) v^{\prime \prime}=\int\left[\Psi(u)-\Psi\left(u\left(s_{0}+\eta\right)\right)\right] v^{\prime \prime}<0 .
$$

This concludes the proof.

With this estimate we can derive a simple but useful convexity property. Remark that translating the set $N_{0}$ results in adding to $u$ functions of the form $v(s):=a \cos s+$ $b \sin s$. Writing the functional in the form (see (2.1))

$$
2 F(u)=\int_{S^{1}} u\left(u+u^{\prime \prime}\right)+M \int_{S^{1}} \Phi\left(\frac{1-u}{M}\right)\left(1+u+u^{\prime \prime}\right),
$$

and differentiating this twice in the direction $v$ we find

$$
2 d^{2} F(u) \cdot v \cdot v=\frac{1}{M} \int_{S^{1}} \Phi^{\prime \prime}\left(\frac{1-u}{M}\right)\left(1+u+u^{\prime \prime}\right) v^{2} .
$$

Since $\Phi^{\prime \prime}((1-u) / M) \geq 0$ whenever $1-M \leq u \leq 1$, with a strict inequality if $u<1$, we find that $F$ is strictly convex with respect to translations of the set $N_{0}$. This proves the following corollary:

Corollary 3.2. If the set $N_{0}$ has an axis of symmetry, then this set is centered in $\Omega$ with respect to this symmetry.

\section{4. $\quad N_{0}$ is a polygon}

In order to prove that $N_{0}$ is a polygon we prove the following, more general, theorem. Consider two numbers $a<b$, and the problem

$$
\inf _{u \in X(a, b)} F(u), \quad \text { where } \quad F(u):=\int_{S^{1}}\left[g(u(s))-u^{\prime 2}(s) h(u(s))\right] d s
$$

where $X(a, b)$ is defined in (2.3). We assume that $g, h \in C^{2}(\mathbb{R})$, and

$$
h(t)>0 \text { for all } t \in(a, b], \quad h(a)=0, \quad g^{\prime}(a)>0 .
$$

Theorem 4.1. Let $u$ be a minimizer of (4.1). Then the support of $u+u^{\prime \prime}$ is a finite subset of $S^{1}$.

This theorem applies directly in our case by setting $a=1-M, b=1, g=\eta, h=\Psi^{\prime}$.

Proof. We assume, to force a contradiction, that there exist sequences $\left(\alpha_{n}\right) \subset$ $(0, \infty),\left(s_{n}\right) \in S^{1}$ such that $\lim \alpha_{n}=0$ and the intersection of the support of 
$\mu:=u+u^{\prime \prime}$ and $\left(s_{n}, s_{n}+\alpha_{n}\right)$ is non-empty. Since $S^{1}$ is compact, the support of $\mu$ has an accumulation point in $S^{1}$; we choose this point to be the origin of $S^{1}$.

Let us first assume that $u(0)>a$; hence $\{u>a\}$ is a neighbourhood of 0 , and there exists a constant $c>0$ such that $h(u)>c$ in this neighbourhood.

For each $\varepsilon>0$, there exists $0<\varepsilon_{1}<\varepsilon_{2}<\varepsilon$ such that

$$
\mu\left(J_{i}\right)>0, \quad i=1,2,3,
$$

for each of the intervals $J_{1}:=\left(0, \varepsilon_{1}\right), J_{2}:=\left[\varepsilon_{1}, \varepsilon_{2}\right), J_{3}:=\left[\varepsilon_{2}, \varepsilon\right)$. For $i=1, \ldots, 3$ and $\varepsilon>0$ given, let $w_{i}$ be the unique solution of the problem

$$
w_{i}(s)+w_{i}^{\prime \prime}(s)=\mu(s) \chi_{J_{i}} \text { for all } s \in(0, \varepsilon), \quad \text { and } w_{i}(0)=w_{i}(\varepsilon)=0 .
$$

Then there exist $\left(\lambda_{i}\right)_{i=1, \ldots, 3}$ such that $v_{\varepsilon}:=\sum_{i=1}^{3} \lambda_{i} w_{i}$ satisfies

$$
0=v_{\varepsilon}^{\prime}(0)=\sum_{i=1}^{3} \lambda_{i} w_{i}^{\prime}(0) \quad \text { and } \quad 0=v_{\varepsilon}^{\prime}(\varepsilon)=\sum_{i=1}^{3} \lambda_{i} w_{i}^{\prime}(\varepsilon) .
$$

We extend this function by zero outside $(0, \varepsilon)$, such that $v_{\varepsilon}^{\prime}$ is continuous at 0 and $\varepsilon$. Hence there exists $\eta_{0}>0$ such that $u+\eta v_{\varepsilon} \in X$ for all $\eta \in\left[-\eta_{0}, \eta_{0}\right]$. Since $u$ solves (4.1), it follows that $F^{\prime \prime}(u) v_{\varepsilon} \cdot v_{\varepsilon} \geq 0$, that is

$$
\begin{aligned}
0 & \leq \int_{S^{1}} g^{\prime \prime}(u) v_{\varepsilon}^{2}-2 h(u) v_{\varepsilon}^{\prime 2}-4 u^{\prime} h^{\prime}(u) v_{\varepsilon}^{\prime} v_{\varepsilon}-u^{\prime 2} h^{\prime \prime}(u) v_{\varepsilon}^{2} \\
& \leq \int_{S^{1}}\left[g^{\prime \prime}(u)+u^{\prime 2} h^{\prime \prime}(u)+2 u^{\prime \prime} h^{\prime}(u)\right] v_{\varepsilon}^{2}-2 h(u) v_{\varepsilon}^{\prime 2}
\end{aligned}
$$

Let us note

such that

$$
c_{1}:=\left\|g^{\prime \prime}(u)+u^{\prime 2} h^{\prime \prime}(u)+2 u^{\prime \prime} h^{\prime}(u)\right\|_{\mathrm{RM}\left(S^{1}\right)}
$$

$$
2 c \int_{0}^{\varepsilon} v_{\varepsilon}^{\prime 2} \leq c_{1}\left\|v_{\varepsilon}\right\|_{L^{\infty}\left(S^{1}\right)}^{2} .
$$

Since by the Sobolev embedding

$$
\left\|v_{\varepsilon}\right\|_{L^{\infty}\left(S^{1}\right)}^{2} \leq c_{0} \varepsilon \int_{0}^{\varepsilon} v_{\varepsilon}^{\prime 2},
$$

where $c_{0}$ does not depend on $\varepsilon$, this contradicts (4.5) for small enough $\varepsilon$.

Hence we have $u(0)=a$. Moreover, from the previous argument we know that the support of $u+u^{\prime \prime}$ is finite in any compact subset of $\left\{s \in S^{1}: u(s)>a\right\}$. Since 0 is not an interior point of the set $\{u=a\}$ we can assume for instance that $u(s)>a$ in a right neighbourhood of 0 , say $\left(0, s_{0}\right)$. Then there exists an infinite decreasing sequence $\left(t_{n}\right)$ with limit 0 and a summable sequence $\left(\alpha_{n}\right) \subset(0, \infty)$, such that the restriction of $u+u^{\prime \prime}$ to $\left(0, s_{0}\right)$ equals $\sum_{n} \alpha_{n} \delta_{t_{n}}$. 
For any given $n \in \mathbb{N}, n>2$, let us define the function $v_{n}$ as follows:

$$
v_{n}(s)= \begin{cases}\sin \left(s-t_{n+1}\right) \sin \left(t_{n-1}-t_{n}\right) & \text { if } s \in\left(t_{n+1}, t_{n}\right) \\ \sin \left(t_{n-1}-s\right) \sin \left(t_{n}-t_{n+1}\right) & \text { if } s \in\left(t_{n}, t_{n-1}\right) \\ 0 & \text { otherwise }\end{cases}
$$

One can easily check that $v_{n}$ is continuous and that the support of $v_{n}+v_{n}^{\prime \prime}$ is the set $\left\{t_{n+1}, t_{n}, t_{n-1}\right\}$. Therefore $u \pm \varepsilon v_{n}$ is admissible for $\varepsilon$ small enough: this implies $F^{\prime}(u) \cdot v_{n}=0$ :

$$
\begin{aligned}
0 & =F^{\prime}(u) \cdot v_{n} \\
& =\int\left[g^{\prime}(u)-\left(u^{\prime}\right)^{2} h^{\prime}(u)\right] v_{n}-2 u^{\prime} h(u) v_{n}^{\prime} \\
& =\int\left[g^{\prime}(u)+\left(u^{\prime}\right)^{2} h^{\prime}(u)+2 u^{\prime \prime} h(u)\right] v_{n} \\
& =\int\left[g^{\prime}(u)+\left(u^{\prime}\right)^{2} h^{\prime}(u)-2 u h(u)\right] v_{n}+2 \int\left(u+u^{\prime \prime}\right) h(u) v_{n}
\end{aligned}
$$

Taking into account that $u+u^{\prime \prime}=\sum_{n} \alpha_{n} \delta_{t_{n}}$, and $v_{n}\left(t_{p}\right)=0$ if $p \neq n$, we find

$$
-g^{\prime}(a)-\left(u^{\prime}(0+)\right)^{2} h^{\prime}(a)=\frac{2 \alpha_{n} h\left(u\left(t_{n}\right)\right) v_{n}\left(t_{n}\right)}{\int v_{n}}+\frac{\int \chi v_{n}}{\int v_{n}}
$$

where

$$
\chi(s):=g^{\prime}(u)-g^{\prime}(a)+\left(u^{\prime}\right)^{2} h^{\prime}(u)-u^{\prime}(0+)^{2} h^{\prime}(a)+2 u h(u) .
$$

Note that $\chi$ has limit 0 in 0 since $u^{\prime}(t+)$ is right continuous everywhere; hence as $n$ goes to infinity, $\int \chi v_{n} / \int v_{n}$ goes to zero. We also have $\int v_{n} / v_{n}\left(t_{n}\right)=\frac{1}{2}\left(t_{n+1}-t_{n-1}\right)+o\left(t_{n}\right)$. On the other hand, $h\left(u\left(t_{n}\right)\right)=\int_{0}^{t_{n}} h^{\prime}(u(t)) u^{\prime}(t) d t \sim h^{\prime}(a) u^{\prime}(0+) t_{n}$ for $n$ large. Hence if we assume that $g^{\prime}(a)+u^{\prime}(0+)^{2} h^{\prime}(a) \neq 0$ we see that there exists $c>0$ such that

$$
\alpha_{n}>c \frac{\tau_{n}+\tau_{n-1}}{\sum_{j=n}^{\infty} \tau_{j}}
$$

where $\tau_{j}:=t_{j-1}-t_{j}$.

However it is known that if $\left(\tau_{n}\right)$ is a positive summable sequence, then $\tau_{n} / \sum_{j \geq n} \tau_{j}$ is not summable. (This comes by considering the step function $k$ such that $k([n, n+1))=\tau_{n}$; then define $K(x):=\int_{x}^{\infty} k$. The sum $\sum\left(\tau_{n} / \sum_{j \geq n} \tau_{j}\right)$ is equivalent to $\int_{n}^{\infty} k / K=[\log (-K)]_{n}^{\infty}=\infty$.) We deduce that $\alpha_{n}$ is not summable, a contradiction.

So we must have

$$
g^{\prime}(a)+u^{\prime}(0+)^{2} h^{\prime}(a)=0 .
$$

Since we assumed $g^{\prime}(a)<0$, we find that $h^{\prime}(a)>0$ and $u^{\prime}(0+) \neq 0$. Passing now to 
the second order derivative, we get, again writing $\tau_{j}:=t_{j-1}-t_{j}$ :

$$
\begin{aligned}
0 \leq & F^{\prime \prime}(u) \cdot v_{n} \cdot v_{n} \\
= & \int\left[g^{\prime \prime}(u)+\left(u^{\prime}\right)^{2} h^{\prime \prime}(u)-2 u h^{\prime}(u)\right] v_{n}^{2} \\
& +2 \int\left(u+u^{\prime \prime}\right) h^{\prime}(u) v_{n}^{2}-2 \int h(u)\left(v_{n}^{\prime}\right)^{2} \\
= & O\left(\left(\tau_{n}+\tau_{n+1}\right)^{5}\right)+2 \alpha_{n} h^{\prime}\left(u\left(t_{n}\right)\right) v_{n}\left(t_{n}\right)^{2}-2 \int h(u)\left(v_{n}^{\prime}\right)^{2} \\
= & o\left(\left(\tau_{n}+\tau_{n+1}\right)^{4}\right)-2 \int h(u)\left(v_{n}^{\prime}\right)^{2}
\end{aligned}
$$

taking into account that $v_{n}\left(t_{n}\right)=\sin \tau_{n} \sin \tau_{n+1}$ and $\lim \alpha_{n}=0$. We recall that $h(u(s)) \sim h^{\prime}(a) u^{\prime}(0+) s$ as $s$ goes to zero; hence

$$
\begin{aligned}
o\left(\left(\tau_{n}+\tau_{n+1}\right)^{4}\right) \geq \int h(u)\left(v_{n}^{\prime}\right)^{2} & \sim h^{\prime}(a) u^{\prime}(0+) t_{n} \int_{t_{n+1}}^{t_{n-1}}\left(v_{n}^{\prime}\right)^{2} \\
& \sim h^{\prime}(a) u^{\prime}(0+) t_{n} \tau_{n} \tau_{n+1}\left(\tau_{n}+\tau_{n+1}\right) .
\end{aligned}
$$

Since $h^{\prime}(a)>0$, we conclude that $u^{\prime}(0+)<0$, and therefore $u$ is not minimal at 0 , a contradiction.

\section{Polygonal functions and symmetry}

We will now prove the following result:

Theorem 5.1. Among all functions whose minimal sets $N_{0}$ are polygons, only the regular ones centered in $\Omega$ (i.e. $N_{0}$ is a regular polygon with center 0$)$ are stable critical points for the functional $F$.

We shall say that a function $u: S^{1} \rightarrow \mathbb{R}$ is polygonal of order $m$ ( $m \geq 1$ integer) if it satisfies:

$$
u+u^{\prime \prime}=\sum_{k=1}^{m} \alpha_{k} \delta_{s_{k}}
$$

in the distributional sense, for some numbers $\alpha_{k}>0$, and different $s_{k} \in S^{1}\left(\delta_{s_{k}}\right.$ is the Dirac measure at $s_{k}$ ). In the following, the numbers $s_{k}$ are assumed to be ordered in $S^{1}$, for instance $-\pi \leq s_{1}<s_{2}<\cdots<s_{m}<\pi$. It is convenient to uses indices modulo $m$, so that for instance $s_{0}:=s_{m}$, and $s_{m+1}:=s_{1}$.

Equation (5.1) implies the compatibility condition

$$
\sum_{k=1}^{m} \alpha_{k} \mathrm{e}^{i s_{k}}=0 .
$$


This can be proved by integrating $u+u^{\prime \prime}$ against cosine or sine functions. Note that, since $u$ is continuous and satisfies (5.1), we have

$$
u_{k}\left(s_{k}\right)=u_{k-1}\left(s_{k}\right) \text { and } u_{k}^{\prime}\left(s_{k}\right)=\alpha_{k}+u_{k-1}\left(s_{k}\right) .
$$

We then have:

$$
2 F(u)=\sum_{k=1}^{m} \alpha_{k} \Psi\left(u\left(s_{k}\right)\right)+\sum_{k=1}^{m} \int_{s_{k}}^{s_{k+1}} \Phi_{M}\left(u_{k}(s)\right) d s,
$$

where $\Phi_{M}(x):=M \Phi\left(\frac{1-x}{M}\right)=\Psi(x)-x$.

The proof of Theorem 5.1 is given in the rest of the paper. Note that the case $m=2$ can be easily achieved right now. This corresponds to a line segment for $N_{0}$; by Corollary 3.2 it is centered in $\Omega$ as claimed. The corresponding function $u$ has the form $u(s)=\mu\left|\cos \left(s-s_{1}\right)\right|$ for some $s_{1} \in S^{1}$.

\subsection{Alternative parameters}

We give some alternative sets of parameters that are useful for new expressions of the functional. Each function $u_{k}$ satisfies $u_{k}+u_{k}^{\prime \prime}=0$ in $\left(s_{k}, s_{k+1}\right)$. Hence we can express it in the form

$$
u_{k}(s)=\mu_{k} \cos \left(s-\theta_{k}\right)
$$

with $\mu_{k} \geq 0$ and $\theta_{k} \in\left(s_{k}-\pi, s_{k}+\pi\right]$. Let us define $a_{k}:=\theta_{k}-s_{k}$ and $b_{k}:=s_{k+1}-\theta_{k}$. We have by assumption

$$
0<\alpha_{k}=u_{k}^{\prime}\left(s_{k}\right)-u_{k-1}^{\prime}\left(s_{k}\right)=\mu_{k} \sin a_{k}+\mu_{k-1} \sin b_{k-1} .
$$

For the value of the functional we get:

$$
\begin{aligned}
2 F(u)= & \sum_{k=1}^{m}\left(\mu_{k} \sin a_{k}+\mu_{k-1} \sin b_{k-1}\right) \Psi\left(u\left(s_{k}\right)\right)+\sum_{k=1}^{m} \int_{-a_{k}}^{b_{k}} \Phi_{M}\left(\mu_{k} \cos t\right) d t \\
= & \sum_{k=1}^{m} \mu_{k} \sin a_{k} \Psi\left(u\left(s_{k}\right)\right)+\sum_{k=1}^{m} \mu_{k} \sin b_{k} \Psi\left(u\left(s_{k+1}\right)\right) \\
& +\sum_{k=1}^{m} \int_{-a_{k}}^{b_{k}} \Phi_{M}\left(\mu_{k} \cos t\right) d t .
\end{aligned}
$$

From their definitions we have

$$
u_{k}\left(s_{k}\right)=\mu_{k} \cos a_{k}=u_{k-1}\left(s_{k}\right)=\mu_{k-1} \cos b_{k-1}
$$

hence

$$
2 F(u)=2 \pi+\sum_{k=1}^{m} K\left(\mu_{k}, a_{k}\right)+K\left(\mu_{k}, b_{k}\right)
$$




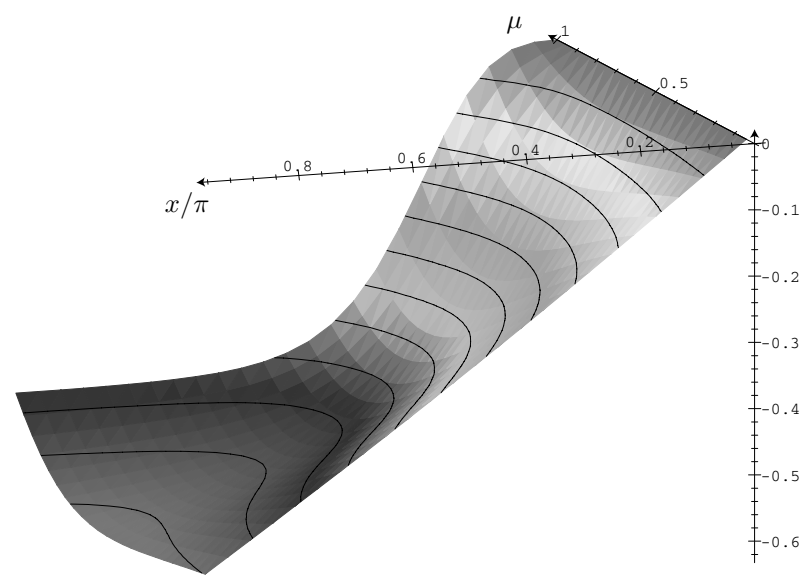

Figure 3: Value of $K(x, \mu)$, for $M=1 / 2$.

where (recall that $\left.\Phi_{M}(x)=\Psi(x)-x\right)$ :

$$
\begin{aligned}
K(\mu, x) & :=\mu \sin x(\Psi(\mu \cos x)-1)+\int_{0}^{x} \Psi(\mu \cos t) d t-x \\
& =\mu \sin x \Psi_{1}(\mu \cos x)+\int_{0}^{x} \Psi_{1}(\mu \cos t) d t
\end{aligned}
$$

where we used the shortcut notation $\Psi_{1}:=\Psi-1$. The additional term $-x$ is permitted since we add $2 \pi$ in $F$ and take into account that $2 \pi=\sum\left(s_{k+1}-s_{k}\right)$, that is

$$
2 \pi=\sum_{k=1}^{m} b_{k}+a_{k}
$$

The previous expression can be used to compute the value of the functional, since the integral can be expressed analytically with respect to $M, \mu$, and $x$, through a complicated expression given in the Appendix. Figure 3 pictures this function for $M=1 / 2$; for other values, the picture is similar.

\subsection{Bounds on the $s_{k}$}

While the formulation of the function $u$ above in terms of the functions $u_{k}$ allows a relative freedom in choosing the values of $s_{k}$, there are limits to this freedom. This is the content of the next Lemma.

Lemma 5.2. Let $u$ be an admissible polygonal function, described by (5.1). For every $k, s_{k}-s_{k-1} \leq \pi$. If $s_{k}-s_{k-1}=\pi$ for some $k$, then $m=2$, and $N_{0}$ is a segment. 
Proof. Suppose that $m \geq 2$, and that $s_{1}-s_{0}>\pi$, so that $\left(s_{0}+2 \pi\right)-s_{1}<\pi$. The operator $A u=-u-u^{\prime \prime}$ satisfies the maximum principle on intervals of length less than $\pi$. Considering the interval $\left[s_{1}, s_{0}+2 \pi\right]$, we note that $u_{1}$ solves $A u_{1}=0$, and $u$ satisfies $A u \leq 0$, and the two are equal on the boundary. It follows that $u_{1} \geq u$, and therefore $u^{\prime}\left(s_{1}-\right)=u_{1}^{\prime}\left(s_{1}\right) \geq u^{\prime}\left(s_{1}+\right)$, with a strict inequality if $u \not \equiv u_{1}$. This contradicts the positivity of $u+u^{\prime \prime}$.

We might note that one could also use a geometrical argument: the section $\left(s_{k-1}, s_{k}\right)$ corresponds to a vertex of the polygon $N_{0}$ (see Figure 2). The values of $s_{k-1}$ and $s_{k}$ correspond to the directions of the sides of the polygon on either side of the vertex, and the corner formed by the two sides has an internal angle of $\pi-\left(s_{k}-s_{k-1}\right)$. If $s_{k}-s_{k-1}>\pi$, then this angle is negative, and therefore $u$ does not correspond to a well-formed set $N_{0}$.

If $s_{1}-s_{0}=\pi$, then $u\left(s_{1}\right)=u\left(s_{0}\right)=0$. Applying a similar argument to the interval $\left[s_{0}+2 \pi, s_{1}\right]$, we find that $u=\mu^{\prime} \sin \left(s-s_{1}\right)$ on $\left[s_{0}+2 \pi, s_{1}\right]$ for some $\mu^{\prime} \in \mathbb{R}$. It follows that $m=2$ and that $N_{0}$ is a segment.

\subsection{Derivative jumps are symmetric}

From now on, we assume that $u$ is a minimizer of $F$.

Lemma 5.3. Suppose that $m \geq 3$. Then for each $k$ we have

$$
u^{\prime}\left(s_{k}+\right)=-u^{\prime}\left(s_{k}-\right)
$$

Proof. We prove the result for $k=1$. In order to shorten notation, we write $z_{k}:=u\left(s_{k}\right)$, and $y_{1}^{+}:=u_{1}^{\prime}\left(s_{1}\right)=u^{\prime}\left(s_{1}+\right), y_{1}^{-}:=u_{0}^{\prime}\left(s_{1}\right)=u^{\prime}\left(s_{1}-\right)$. Note that since $m \geq 3$, by the previous Lemma, $s_{2}-s_{1}<\pi$ and $s_{1}-s_{0}<\pi$.

Let $\varepsilon \neq 0$ be given. We define $d_{\varepsilon}:=\left(u_{1}\left(s_{1}+\varepsilon\right)-u_{0}\left(s_{1}+\varepsilon\right)\right) / \varepsilon$ and $d_{0}:=\left(y_{1}^{+}-y_{1}^{-}\right)$; one easily checks that $d_{\varepsilon}=d_{0}+o(1)$ for small $\varepsilon$ since $u_{1}\left(s_{1}\right)=z_{1}=u_{0}\left(s_{1}\right)$.

Let us consider the function $v_{0}$ such that $v_{0}+v_{0}^{\prime \prime}=0, v_{0}\left(s_{2}\right)=0$ and $v_{0}\left(s_{1}\right)=d_{0}$. For $\varepsilon \neq 0$ we also define $v_{\varepsilon}$ by $v_{\varepsilon}+v_{\varepsilon}^{\prime \prime}=0, v_{\varepsilon}\left(s_{2}\right)=0$ and $v_{\varepsilon}\left(s_{1}+\varepsilon\right)=d_{\varepsilon}$. Here again, we see that $v_{\varepsilon} \equiv v_{0}+o(1)$ for $\varepsilon$ small enough.

We consider the function $u^{\varepsilon}$ defined as follows:

$$
u^{\varepsilon}(s)= \begin{cases}u_{0}(s) & \text { if } s \in\left(s_{0}, s_{1}+\varepsilon\right), \\ u_{1}(s)-\varepsilon v_{\varepsilon}(s) & \text { if } s \in\left(s_{1}+\varepsilon, s_{2}\right) \\ u(s) & \text { otherwise }\end{cases}
$$

(Observe that $u^{\varepsilon}$ is continuous by the definition of $v_{\varepsilon} \cdot$ ) We note that $u^{\varepsilon}$ is admissible for any small enough $\varepsilon$. Hence $F\left(u^{\varepsilon}\right) \geq F(u)$ since $u$ is a minimizer. We note that 
by $(2.4)$

$$
\begin{aligned}
2 F\left(u^{\varepsilon}\right)= & \int_{s_{0}}^{s_{1}+\varepsilon}(\Psi-\mathrm{id})\left(\mathrm{u}_{0}\right)+\int_{\mathrm{s} 1+\varepsilon}^{\mathrm{s}_{2}}(\Psi-\mathrm{id})\left(\mathrm{u}_{1}-\varepsilon \mathrm{v}_{\varepsilon}\right) \\
& +\int_{s_{2}+0}^{s_{0}+2 \pi}\left[\Psi(u)\left(1+u+u^{\prime \prime}\right)-u\right] \\
& +\Psi\left(u^{\varepsilon}\left(s_{1}+\varepsilon\right)\right)\left[u_{1}^{\prime}\left(s_{1}+\varepsilon\right)-\varepsilon v_{\varepsilon}^{\prime}\left(s_{1}+\varepsilon\right)-u_{0}^{\prime}\left(s_{1}+\varepsilon\right)\right] \\
& +\Psi\left(u\left(s_{2}\right)\right)\left[u_{2}^{\prime}\left(s_{2}\right)-u_{1}^{\prime}\left(s_{2}\right)+\varepsilon v_{\varepsilon}^{\prime}\left(s_{2}\right)\right] .
\end{aligned}
$$

We recall that $u_{0}\left(s_{1}\right)=z_{1}=u_{1}\left(s_{1}\right)$; hence $\int_{s_{1}}^{s_{1}+\varepsilon} \Psi\left(u_{0}\right)-\Psi\left(u_{1}\right)=o(\varepsilon)$. Moreover, since $u_{i}+u_{i}^{\prime \prime}=0$, we have $u_{1}^{\prime}\left(s_{1}+\varepsilon\right)-u_{0}^{\prime}\left(s_{1}+\varepsilon\right)=y_{1}^{+}-y_{1}^{-}+O\left(\varepsilon^{2}\right)$. We also have:

$$
\Psi\left(u^{\varepsilon}\left(s_{1}+\varepsilon\right)\right)=\Psi\left(u_{0}\left(s_{1}+\varepsilon\right)\right)=\Psi\left(z_{1}\right)+\varepsilon \Psi^{\prime}\left(z_{1}\right) y_{1}^{-}+o(\varepsilon) .
$$

This yields

$$
\begin{aligned}
0 \leq & F\left(u^{\varepsilon}\right)-F(u) \\
\leq & o(\varepsilon)-\varepsilon \int_{s_{1}}^{s_{2}}\left[\Psi^{\prime}\left(u_{1}\right)-1\right] v_{0}+\varepsilon \Psi^{\prime}\left(z_{1}\right) y_{1}^{-}\left(y_{1}^{+}-y_{1}^{-}\right) \\
& -\varepsilon \Psi\left(z_{1}\right) v_{0}^{\prime}\left(s_{1}\right)+\varepsilon \Psi\left(z_{2}\right) v_{0}^{\prime}\left(s_{2}\right) .
\end{aligned}
$$

Since this holds for any $\varepsilon$ small enough (positive or negative), we get

$$
\Psi^{\prime}\left(z_{1}\right) y_{1}^{-}\left(y_{1}^{-}-y_{1}^{+}\right)=\int_{s_{1}}^{s_{2}}\left[\Psi^{\prime}\left(u_{1}\right)-1\right] v_{0}-\Psi\left(z_{1}\right) v_{0}^{\prime}\left(s_{1}\right)+\Psi\left(z_{2}\right) v_{0}^{\prime}\left(s_{2}\right) .
$$

We briefly consider the possibility that $\Psi^{\prime}\left(z_{1}\right)=0$. The integral in (5.9) is negative, so that we then find

$$
-\Psi\left(z_{1}\right) v_{0}^{\prime}\left(s_{1}\right)+\Psi\left(z_{2}\right) v_{0}^{\prime}\left(s_{2}\right) \geq 0 .
$$

A simple argument using the concaveness of $v_{0}$ and the monotonicity of $\Psi$ shows that this inequality is not satisfied. This proves the Lemma if $\Psi^{\prime}\left(z_{1}\right)=0$.

We continue with the case $\Psi^{\prime}\left(z_{1}\right)>0$, and consider the variation which is analogous to the one above, but with $s_{2}$ replaced by $s_{0}$. Beginning with the function $w_{0}$ such that $w_{0}+w_{0}^{\prime \prime}=0, w_{0}\left(s_{0}\right)=0, w_{0}\left(s_{1}\right)=d_{0}$, and operating exactly the same way in the interval $\left(s_{0}, s_{1}\right)$ we get similarly:

$$
\Psi^{\prime}\left(z_{1}\right) y_{1}^{+}\left(y_{1}^{-}-y_{1}^{+}\right)=\int_{s_{1}}^{s_{0}}\left[\Psi^{\prime}\left(u_{0}\right)-1\right] w_{0}-\Psi\left(z_{1}\right) w_{0}^{\prime}\left(s_{1}\right)+\Psi\left(z_{0}\right) w_{0}^{\prime}\left(s_{0}\right)
$$

Finally let us consider the function

$$
\tilde{u}(s):= \begin{cases}u_{0}-\varepsilon w_{0}(s) & \text { if } \quad s \in\left(s_{0}, s_{1}\right), \\ u_{1}-\varepsilon v_{0}(s) & \text { if } s \in\left(s_{1}, s_{2}\right), \\ u(s) & \text { otherwise. }\end{cases}
$$


Here we also have an admissible function, since $v_{0}\left(z_{1}\right)=w_{0}\left(z_{1}\right)$. Applying a first order variation argument again, we find that the right-hand side of (5.9) and (5.10) are just opposite. This yields

$$
\Psi^{\prime}\left(z_{1}\right)\left[\left(y_{1}^{+}\right)^{2}-\left(y_{1}^{-}\right)^{2}\right]=0 .
$$

Since $y_{1}^{-} \neq y_{1}^{+}$by assumption $\left(u^{\prime}\right.$ is not continuous at $\left.s_{1}\right)$, and $\Psi^{\prime}\left(z_{1}\right)>0$, the proof is complete.

\subsection{Proof of Theorem 5.1}

From Lemma 5.3 we have for each $k, \mu_{k} \sin a_{k}=\mu_{k-1} \sin b_{k-1}$. Combining with (5.5) we get

$$
\mu_{k}=\mu_{k-1} \text { and } a_{k}=b_{k-1}
$$

in that case. Hence the functional can be expressed with respect to the unique parameter $\mu$ (the common value of all $\mu_{k}$ ) and to the $a_{k}$, subject to the condition

$$
\sum_{i=1}^{m} a_{k}=\pi .
$$

The derivative jump at $s_{k}$ is given by $\mu_{k} \sin a_{k}+\mu_{k-1} \sin b_{k-1}=2 \mu \sin a_{k}$, and the convexity condition $u+u^{\prime \prime} \geq 0$ therefore implies that $a_{k} \in[0, \pi]$. The functional itself is just $\sum K\left(\mu, a_{k}\right)$, hence for the minimizer there exists a Lagrange multiplier $\gamma$ such that

$$
\frac{\partial F}{\partial a_{k}}=K_{x}^{\prime}\left(\mu, a_{k}\right)=\gamma
$$

for all $k$. On the other hand we have from (5.7):

$$
K_{x}^{\prime}(\mu, x)=(1+\mu \cos x) \Psi_{1}(\mu \cos x)-\mu^{2} \sin ^{2} x \Psi^{\prime}(\mu \cos x) .
$$

We now have to prove that all $a_{k}$ are equal. We first prove an intermediate result:

$$
K_{x}^{\prime}\left(\mu, a_{k}\right)=\gamma \leq K_{x}^{\prime}(\mu, 0) .
$$

Indeed, consider a small number $\varepsilon>0$, and a function $u^{\varepsilon}$ characterized by $(m+1)$ sides with parameters $a_{k}^{\varepsilon}=b_{k-1}^{\varepsilon}=a_{k}+\delta a_{k}$, for $k=1, \ldots, m$, and $a_{m+1}^{\varepsilon}=b_{m}^{\varepsilon}=\varepsilon$; $\mu_{k}^{\varepsilon}=\mu$ for $k=1, \ldots,(m+1)$. This function satisfies the constraints if we assume that $\sum \delta a_{k}=-\varepsilon$. We then have

$$
\begin{aligned}
F\left(u^{\varepsilon}\right)-F(u) & =\sum_{k=1}^{m}\left[K\left(\mu, a_{k}^{\varepsilon}\right)-K\left(\mu, a_{k}\right)\right]+K(\mu, \varepsilon) \\
& =\sum_{k=1}^{m} K_{x}^{\prime}\left(\mu, a_{k}\right) \delta a_{k}+\varepsilon K^{\prime}(\mu, 0)+o(\varepsilon) .
\end{aligned}
$$

We already know from (5.11) that $K_{x}^{\prime}\left(\mu, a_{k}\right)=\gamma$ for all $k$. Since we have $F\left(u^{\varepsilon}\right) \geq F(u)$ for all $\varepsilon>0$, we get (5.13). 


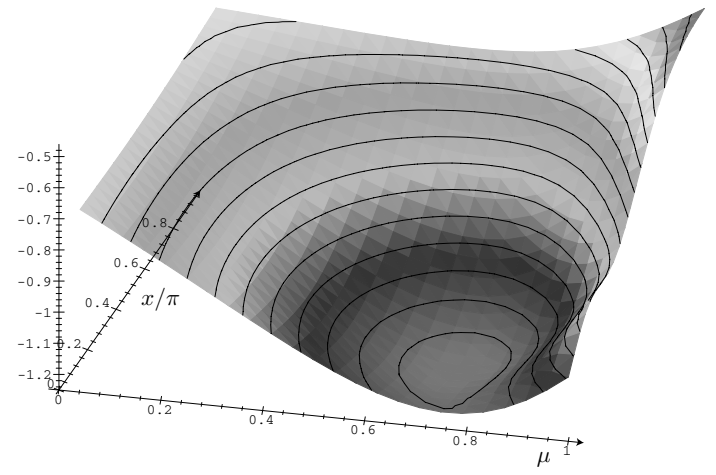

Figure 4: Value of $(m-1) K(\mu, x)+K(\mu, \pi-x(m-1))$ for $M=1 / 2, m=4$.

The function $x \mapsto K_{x}^{\prime}(\mu, x)$ is explicitly given by (5.12). We can normalize the function $K$ by subtracting $x K_{x}^{\prime}(\mu, 0)$ : note that this adds a constant to $F$, so that this does not change the nature of the problem; for this normalized function, still noted $K$, we have $K_{x}^{\prime}(\mu, 0)=0$. From (5.13), the only values of interest in the following are those such that $K_{x}^{\prime} \leq 0$; this corresponds to an interval $[0, \bar{x}]$. One can easily check the following properties: if $\mu$ is small, then $K_{x}^{\prime}$ is an increasing function on $(0, \pi]$; if $\mu$ is larger than a critical value (depending on $M$ ), then on $(0, \pi]$ this function is strictly decreasing, then increasing. (This last property is proved in Appendix B.)

From (5.13) we see that $\mu$ must be larger than the critical value indicated before. In that case, the function $K_{x}^{\prime}(\mu, \cdot)$ attains a strict minimum $x_{0} \in(0, \pi)$ and the equation $K_{x}^{\prime}(\mu, x)=\gamma_{1} \leq K_{x}^{\prime}(\mu, 0)$ has two solutions $x_{1}, x_{2}$ satisfying

$$
0 \leq x_{1}<x_{0}<x_{2}<\pi \text { and } K_{x x}^{\prime \prime}\left(\mu, x_{1}\right)<0<K_{x x}^{\prime \prime}\left(\mu, x_{2}\right)
$$

(except in the special case $K_{x}^{\prime}(\mu, x)=K_{x}^{\prime}\left(\mu, x_{0}\right)$ ). That gives two potential values for the $a_{k}$.

It easy to check that at most one $a_{k}$ can be equal to $x_{1}$. Indeed, we just have to consider a small variation in the form $\tilde{a}_{1}=a_{1}+\varepsilon, \tilde{a}_{2}=a_{2}-\varepsilon$, and we find $K_{x x}^{\prime \prime}\left(\mu, a_{1}\right)+K_{x x}^{\prime \prime}\left(\mu, a_{2}\right) \geq 0$ for a minimizer.

Hence we have to choose between two possibilities: either all $a_{k}$ are equal to $\pi / m$ (regular polygon), or all of them except one are equal (say to a number $x=x_{2}$ ), and the other one $\left(x_{1}=\pi-(m-1) x\right)$ is different; $x_{1}$ and $x_{2}$ satisfy (5.14) and $K_{x}^{\prime}\left(\mu, x_{1}\right)=K_{x}^{\prime}\left(\mu, x_{2}\right)$. We can catch both possibilities by minimizing

$$
G(\mu, m, x):=(m-1) K(\mu, x)+K(\mu, \pi-(m-1) x)
$$

with respect to $\mu \in(0,1), m \in \mathbb{N}$, and $x \in I_{m}$ given later. If the minimum satisfies $x=\pi / m$, then all $a_{k}$ are equal; alternatively, if $x \neq \pi / m$, then the $a_{k}$ are different.

The set $I_{m}$ of admissible values of $x$ is given by $I_{m}=[\pi / m, \pi /(m-1))$. This results from the following argument. Clearly $\pi / m \in I_{m}$; we do not need to take into account values $x<\pi / m$, since by (5.14) $x_{1}=\pi-(m-1) x \leq x_{2}=x$; and $x \geq \pi /(m-1)$ 
corresponds to $x_{1} \leq 0$, which is also excluded, as mentioned above. Moreover we can restrict, from (5.14), to those values of $(\mu, m, x)$ such that $K_{x x}^{\prime \prime}(\mu, x)>0$. Since $K_{x x}^{\prime \prime}$ vanishes at most once in $(0, \pi)$ (cf. Appendix B), it is positive at least at one end of $I_{m}$. That is either

$$
\begin{aligned}
& K_{x x}^{\prime \prime}(\mu, \pi / m)>0 \\
& \text { or } K_{x x}^{\prime \prime}(\mu, \pi / m) \leq 0 \text { and } K_{x x}^{\prime \prime}(\mu, \pi /(m-1))>0 .
\end{aligned}
$$

The function $G$ is pictured for $M=1 / 2, m=4$, in Figure 4; there is a clear minimum in this case with $x=\pi / 4$, corresponding to a regular polygon for $N_{0}$. Notice that $G$ is not convex with respect to $x$ (though we already know that it is convex with respect to $\mu$ ).

If ( $\mu, m, x)$ minimizes $G$, then $x$ also minimizes the restricted function $x \mapsto G(\mu, m, x)$ for these specific values of $\mu$ and $m$. With this remark in mind we fix $\mu$ and $m$ for the remainder of the proof, still assuming (5.15).

We will use the following lemma:

Lemma 5.4. Let $m \geq 2$ be an integer. The equation $G_{x}^{\prime}(\mu, m, x)=0$ has at most two solutions for $x$ in the interval $I_{m}=\left[\frac{\pi}{m}, \frac{\pi}{m-1}\right)$. Moreover, if (5.15) holds with $K_{x x}^{\prime \prime}(\mu, \pi / m) \leq 0$, this equation has only one solution.

The proof of the lemma relies heavily on an analytic expression of $G_{x}^{\prime}$; it is given in Appendix B. We continue here with the proof of the theorem.

One of the solutions is $x=\pi / m$; if $K_{x x}^{\prime \prime}(\mu, \pi / m) \leq 0$, then it is the only one from the lemma, but it is not a minimizer: this is a contradiction since we assumed the opposite about $(m, \mu)$.

Let us now assume that $K_{x x}^{\prime \prime}(\mu, \pi / m)>0$. Then $G$ attains a strict local minimum at $x=\pi / m$. Therefore, if $G_{x}^{\prime}$ vanishes only once in $I_{m}$, then $G$ is increasing on $I_{m}$, and the Theorem is proved.

Alternatively, if $G_{x}^{\prime}$ vanishes at some other point $x^{\prime} \in I_{m}\left(x^{\prime} \neq \pi / m\right)$, we deduce that $G_{x x}^{\prime \prime}\left(\mu, m, x^{\prime}\right) \leq 0$ since $G$ is an analytic function of $x$. Hence $x^{\prime}$ is a local maximum of $G$ (or possibly an inflexion point if $G_{x x}^{\prime \prime}=0$ ). So in any case, $G$ does not have any other minimum in the interior of $I_{m}$.

This ends the proof of Theorem 5.1.

\section{Appendix A: Analytic expression of the functional}

We give here some indication on the way to compute explicitly the integral in $K$, or more precisely:

$$
I(M, \mu, x):=-M^{-2} \int_{0}^{x} \Psi_{1}(\mu \cos t) d t=\int_{0}^{x} \frac{(1-\mu \cos t)}{M^{2}+(1-\mu \cos t)^{2}} d t .
$$

Even with Maple, this does not come straightforwardly since the integrand is quite complicated. Anyway, one can see that it depends only on $2 \cos t=e^{i t}+e^{-i t}$. So we 
can look for a primitive function in the form $J\left(e^{i x}\right)$. We then have

$$
i z J^{\prime}(z)=2 z \frac{2 z-\mu\left(1+z^{2}\right)}{4 z^{2} M^{2}+\left(2 z-\mu\left(1+z^{2}\right)\right)^{2}} .
$$

Since the roots of the denominator can be expressed easily, we can find $J$. The more complicated part is to go back to a real function. At the end, we get

$$
I=c_{l} \arctan \left(\frac{\sin x \cos \alpha}{\cos x-\sin \alpha \cos \beta}\right)+c_{r} \log \left(\frac{1-\sin \alpha \cos (\beta-x)}{1-\sin \alpha \cos (\beta+x)}\right)
$$

where

$$
c_{l}:=\frac{\cos \alpha \cos ^{2} \beta}{\cos ^{2} \alpha+\sin ^{2} \alpha \sin ^{2} \beta} \quad \text { and } \quad c_{r}:=\frac{1}{4} \frac{\sin 2 \beta}{\cos ^{2} \alpha+\sin ^{2} \alpha \sin ^{2} \beta}
$$

and the parameters $\alpha, \beta$ are linked to $M, \mu$ by the relations

$$
\begin{aligned}
\sqrt{2} \cos \alpha=\sqrt{1-M^{2}-\mu^{2}+\nu}, & \sqrt{2} \sin \alpha=\sqrt{M^{2}+\mu^{2}+1-\nu}, \\
\sqrt{2} \mu \cos \beta & =\sqrt{M^{2}+\mu^{2}+1-\nu}, \quad \sqrt{2} \mu \sin \beta=\sqrt{\mu^{2}-M^{2}-1+\nu}
\end{aligned}
$$

with

$$
\nu:=\sqrt{\left(1+M^{2}\right)^{2}-2 \mu^{2}+2 \mu^{2} M^{2}+\mu^{4}} .
$$

\section{Appendix B. Proof of Lemma 5.4}

From formula (5.12), $K_{x}^{\prime}$ depends on $x$ only by the value of $\cos x$. More precisely, we have $K_{x}^{\prime}(\mu, x)=Q(\cos x)+$ constant (the constant was introduced by the normalization) where

$$
Q(z):=\frac{2 \mu^{2} z^{2} M^{4}-\mu^{2} M^{4}+M^{2}(1-\mu z)^{2}\left(1+\mu^{2}\right)+(1-\mu z)^{4}}{\left(M^{2}+(1-\mu z)^{2}\right)^{2}} .
$$

With this notation, the derivative of $G$ is

$$
G_{x}^{\prime}(m, x)=(m-1)[Q(\cos x)-Q(-\cos (m-1) x)]
$$

Let us first assume that $m=2$. Then $G_{x}^{\prime}(2, x)=0$ for $x \neq \pi / 2$ implies $Q(z)=$ $Q(-z)$ for some $z \in(0,1]$. Let us prove that in fact $Q(z)<Q(-z)$ for all $z \in(0,1]$. From the previous expression of $Q$, we get $Q(z)-Q(-z)=-z \mu M^{2} P\left(z^{2}\right) /[D(z) D(-z)]$ where $D(z)$ is the (positive) denominator in the expression of $Q$, and

$$
\begin{aligned}
P(Z):= & \left(\mu^{4}-4 \mu^{4} M^{2}-\mu^{6}\right) Z^{2} \\
& +\left(-2 \mu^{2}-4 \mu^{2} M^{4}+2 \mu^{4} M^{2}+2 \mu^{4}-2 \mu^{2} M^{2}\right) Z \\
& +2 M^{2}+1+3 \mu^{2} M^{4}+2 \mu^{2} M^{2}+M^{4}-\mu^{2} .
\end{aligned}
$$


So we have to prove that $P(Z)>0$ for all $Z \in[0,1]$. Note that this inequality holds at 0 and 1 :

$$
\begin{aligned}
& P(0)=2 M^{2}+3 \mu^{2} M^{4}+2 \mu^{2} M^{2}+M^{4}+1-\mu^{2}>0, \\
& P(1)=\left(1-\mu^{2}\right) M^{4}+2 M^{2}\left(1-\mu^{4}\right)+\left(1-\mu^{2}\right)^{3}>0 .
\end{aligned}
$$

The derivative of $P$ vanishes at $Z_{m}=A / B$ where

$$
A:=2 M^{4}+\left(1-\mu^{2}\right)\left(1+M^{2}\right)>0
$$

(recall that $\mu \in(0,1)$ ) and

$$
B:=-\mu^{2}\left(-1+4 M^{2}+\mu^{2}\right) .
$$

We observe that

$$
A-B=2 M^{4}+3 \mu^{2} M^{2}+M^{2}+1-2 \mu^{2}+\mu^{4}>0
$$

hence either $B<0$ and $Z_{m}<0$, or $B>0$ and $Z_{m}>1$ since $A>B$; in either case, $P$ does not have a local extremum in [0,1]. It follows that $P>0$ in $[0,1]$, and this proves the lemma for $m=2$.

Let us now assume that $m \geq 3$, hence $\cos (m-1) x \leq 0$ in $I_{m}$. Here $G_{x}^{\prime}(m, x)=0$ with $x \neq \pi / m, x \in I_{m}$, is equivalent to the existence of a solution of $Q\left(z_{1}\right)=Q\left(z_{2}\right)$, with $z_{1}$ and $z_{2}$ in $[0,1], z_{1} \neq z_{2}$. We will prove here that in [0,1], either $Q$ is monotonic or $Q^{\prime}$ has only one root. In the first case, $Q\left(z_{1}\right)=Q\left(z_{2}\right)$ implies $z_{1}=z_{2}$. In the second case, if a solution $x \neq \pi / m$ of $G_{x}^{\prime}=0$ exists, it is unique since the functions $\cos x$ and $\cos (m-1) x$ are monotonic in $I_{m}$. This will prove the first assertion of the lemma.

So we have to count the number of zeroes of $Q^{\prime}$ in $[0,1]$. We have

$$
Q^{\prime}(z)=\frac{2 \mu M^{2} N(z)}{\left(M^{2}+(1-\mu z)^{2}\right)^{3}}
$$

with

$$
\begin{aligned}
N(z):= & \left(-\mu^{5}+\mu^{3}-2 \mu^{3} M^{2}\right) z^{3}+\left(3 \mu^{4}-3 \mu^{2}\right) z^{2} \\
& +\left(3 \mu^{3} M^{2}-3 \mu^{3}+3 \mu+3 \mu M^{2}+2 \mu M^{4}\right) z+\mu^{2}-3 \mu^{2} M^{2}-1-M^{2} .
\end{aligned}
$$

We only have to count the zeroes of $N$ in $[0,1]$. We will use the Theorem of Fourier and BUDAN [1, page 173], an improved version of the Dirichlet criterion, which asserts that the number of zeroes of the polynomial $N$ in $[0,1]$ cannot exceed $|\nu(0)-\nu(1)|$, where $\nu(z)$ is the number of changes of signs in the list

$$
\ell(z):=\left[N(z), N^{\prime}(z), N^{\prime \prime}(z), N^{\prime \prime \prime}(z)\right] .
$$

Now we have

$$
\begin{aligned}
\ell(0)= & {\left[\mu^{2}-1-3 \mu^{2} M^{2}-M^{2}, 3 \mu\left(\mu^{2} M^{2}+1-\mu^{2}+M^{2}\right)+2 \mu M^{4},\right.} \\
& \left.6 \mu^{2}\left(\mu^{2}-1\right), 6 \mu^{3}\left(1-\mu^{2}-2 M^{2}\right)\right] .
\end{aligned}
$$


We recall that $\mu \in(0,1)$, so the signs in this list are $\left[-,+,-, \sigma_{0}\right]$ where $\sigma_{0}$ is the sign of $1-\mu^{2}-2 M^{2}$.

Next we have

also

$$
N^{\prime}(1)=\mu\left[3 M^{2}\left(1-\mu^{2}\right)+2 M^{4}+3(\mu+1)(1-\mu)^{3}\right]>0,
$$

$$
N^{\prime \prime}(1)=6 \mu^{2}\left[-2 \mu M^{2}-(\mu+1)(\mu-1)^{2}\right]<0,
$$

and obviously $N^{\prime \prime \prime}(1)=N^{\prime \prime \prime}(0)$. The signs in the list $\ell(1)$ are $\left[\sigma_{1},+,-, \sigma_{0}\right]$ where $\sigma_{1}$ is the sign of $N(1)$. We see that $\nu(0)=\nu(1)$ if $\sigma_{1}<0$, and $\nu(0)=1+\nu(1)$ if $\sigma_{1}>0$. We get $|\nu(0)-\nu(1)| \leq 1$; this ends the proof of the first assertion of the lemma.

We now turn to the second assertion, assuming that $Q$ is not monotonic (otherwise there is nothing to prove), and assuming (5.15) with $K_{x x}^{\prime \prime}(\mu, \pi / m) \leq 0$, or equivalently $\pi / m \leq x_{0}<\pi /(m-1)$ (we recall that $x_{0}$ is the root of $K_{x x}^{\prime \prime}$; we have $z_{0}=\cos x_{0}$, where $z_{0}$ satisfies $\left.N\left(z_{0}\right)=0\right)$. Notice that, from our previous study, we must have $N(1) \geq 0$ in order to have a root for $N$ in $(0,1]$, that is

$$
M^{2} \geq M_{1}^{2}:=\frac{(1-\mu)^{2}(1+\mu)}{2 \mu} .
$$

Moreover, $Q$ is decreasing in $\left[0, z_{0}\right]$ and increasing in $\left[z_{0}, 1\right]$.

We will prove later on that

$$
\text { for all } h \in\left(0, z_{0}\right], \quad Q\left(z_{0}+h\right)>Q\left(z_{0}-h\right) .
$$

Let us explain first how we can deduce the second assertion of the lemma from this. Let $x \in(\pi / m, \pi /(m-1))$ be given, and $x^{\prime}:=\pi-(m-1) x<\pi / m \leq x_{0}$. If $x \leq x_{0}$, then $\cos x^{\prime}>\cos \frac{\pi}{m}>\cos x \geq z_{0}$ and therefore $Q(\cos x)<Q\left(\cos x^{\prime}\right)$ since $Q$ is increasing in $\left[z_{0}, 1\right]$. This implies $K_{x}^{\prime}(\mu, x) \neq 0$.

Let us now assume that $x>x_{0}$. Since $m \geq 3$ we have

$$
1+\cos \frac{\pi}{m-1} \geq 2 \cos \frac{\pi}{m} \text {. }
$$

(Indeed equality occurs for $m=3$, and for $m \geq 4$ we have

$$
\begin{aligned}
1+\cos \frac{\pi}{m-1}-2 \cos \frac{\pi}{m} & >1+\left(1-\frac{\pi^{2}}{2(m-1)^{2}}\right)-2\left(1-\frac{\pi^{2}}{2 m^{2}}+\frac{\pi^{4}}{24 m^{4}}\right) \\
& =\frac{\pi^{2}}{2 m^{2}}\left(2-\frac{m^{2}}{(m-1)^{2}}-\frac{\pi^{2}}{6 m^{2}}\right) \\
& \geq 0 .)
\end{aligned}
$$

Now (5.18) implies

$$
\cos x+\cos x^{\prime}=\cos x-\cos (m-1) x>2 \cos \frac{\pi}{m} \geq 2 z_{0} ;
$$


this follows from remarking that the map $y \mapsto \cos y-\cos (m-1) y-2 \cos \frac{\pi}{m}$ is concave in $I_{m}$ (the second - order derivative is negative), vanishes at $y=\pi / m$, and attains the nonnegative value $1+\cos \frac{\pi}{m-1}-2 \cos \frac{\pi}{m}$ at $y=\pi /(m-1)$. Therefore, if we write $\cos x$ in the form $z_{0}-h\left(h \in\left(0, z_{0}\right]\right)$, we have $\cos x^{\prime}>z_{0}+h$, hence $Q\left(\cos x^{\prime}\right)>Q\left(z_{0}+h\right)>$ $Q\left(z_{0}-h\right)=Q(\cos x)$ using (5.17). This proves that $K^{\prime}(\mu, x) \neq 0$ as claimed in the lemma.

We finally need to prove (5.17). A computation of $\delta Q:=Q\left(z_{0}+h\right)-Q\left(z_{0}-h\right)$ gives a complicated formula for a general $z_{0}$. But since $z_{0}$ satifies $Q^{\prime}\left(z_{0}\right)=0$, we have $\delta Q \in O\left(h^{3}\right)$ for small $h$. An exact computation gives the formula

$$
\delta Q:=4 M^{2} \mu^{3} h^{3} \frac{\mu^{2} A h^{2}+4 M^{2} B}{D\left(z_{0}+h\right) D\left(z_{0}-h\right)}
$$

where

$$
\begin{aligned}
& A:=\mu z_{0}-\mu^{3} z_{0}-2 M^{2} \mu z_{0}+4 M^{2}-1+\mu^{2} \\
& B:=-2 \mu^{2} z_{0}^{2}+M^{2} \mu z_{0}+2 \mu^{3} z_{0}+M^{2}+2 \mu z_{0}-2 \mu^{2} .
\end{aligned}
$$

Hence it is sufficient to prove that $A>0, B>0$ for all $(M, \mu)$ such that $\mu \in(0,1)$ and $M$ satisfies (5.16); here $z_{0}$ is implicitly determined with respect to $(\mu, M)$ by the equation $N\left(z_{0}\right)=0$, that is:

$$
\begin{aligned}
0= & \left(\mu^{3}-2 M^{2} \mu^{3}-\mu^{5}\right) z_{0}^{3}+\left(-3 \mu^{2}+3 \mu^{4}\right) z_{0}^{2} \\
& +\left(3 \mu+3 \mu M^{2}+3 M^{2} \mu^{3}-3 \mu^{3}+2 \mu M^{4}\right) z_{0}-3 M^{2} \mu^{2}-1+\mu^{2}-M^{2} .
\end{aligned}
$$

It is sufficient to prove that $A \cdot B \neq 0$ for all values of $(\mu, M)$, since one can easily check that $A>0, B>0$ for some of these values: for instance, as $\mu \rightarrow 1$, we have $z_{0} \rightarrow 1$, and then $A \rightarrow 2 M^{2}>0, B \rightarrow 2 M^{2}$.

In order to take into account (5.16), we write $M^{2}$ in the form $M_{1}^{2} / t$, hence $t \in(0,1]$. Then we have $A \cdot B=(1-\mu)\left(1-\mu^{2}\right) C$ with (we write $z$ instead of $z_{0}$ from now on):

$$
\begin{aligned}
C:= & \left(-\mu^{5}-5 \mu^{5} t-4 \mu^{5} t^{2}+\mu^{3}-4 \mu^{4} t^{2}-11 \mu^{3} t+\mu^{4}-\mu^{2}\right) z^{2} \\
& +\left(8 \mu^{3} t^{2}+18 \mu^{4} t-3 \mu^{2}+3 \mu^{4}+3 \mu+8 \mu^{4} t^{2}-3 \mu^{3}+14 \mu^{2} t\right) z \\
& +2 \mu^{3}-4 \mu^{3} t^{2}-4 \mu^{2} t^{2}-2 \mu-2 \mu^{2}+2-13 \mu^{3} t-3 \mu t
\end{aligned}
$$

Since $z$ is a root of $N$, we also have

$$
0=2 \mu(\mu z-1)^{3} t^{2}+(\mu-1)(\mu z-1)\left(2 \mu^{2} z^{2}+2 \mu z-3 \mu^{2}-1\right) t-(\mu+1)(\mu-1)^{3} z
$$

This is an equation of degree 2 in $t$. Substituting $t=0$ gives the value $(\mu+1)(1-\mu)^{3} z \geq$ 0 , and $t=(1-\mu) /(1-\mu z)$ gives the value $(\mu-1)^{2}(z-1)\left(2 \mu^{2} z+1+2 \mu+3 \mu^{2}\right) \leq 0$, hence there is always a root in $\left[0, \frac{1-\mu}{1-\mu z}\right]$, the other one being negative. This root can be expressed as follows:

$$
t_{1}:=(1-\mu) \frac{2 \mu^{2} z^{2}+2 \mu z-3 \mu^{2}-1+r}{4 \mu(\mu z-1)^{2}}
$$


where $r:=\sqrt{4 \mu^{4} z^{4}+8 \mu^{3} z^{3}-4 \mu^{4} z^{2}-20 \mu^{3} z+4 \mu z+9 \mu^{4}+6 \mu^{2}+1-8 \mu^{2} z^{2}}$. Substituting $t=t_{1}$ in $C$, we get $4 C=(\alpha r+\beta)(1-\mu) /(1-\mu z)^{2}$ with

$$
\begin{aligned}
\alpha:= & 22 \mu^{3} z-9 \mu^{2}-15 \mu^{2} z^{2}-\mu^{4} z^{2}+10 \mu z-6 \mu^{4}-1, \\
\beta:= & 2 \mu^{6} z^{4}-\mu^{6} z^{2}+18 \mu^{6}+22 \mu^{5} z^{3}-86 \mu^{5} z+76 \mu^{4} z^{2}+33 \mu^{4}-34 \mu^{4} z^{4} \\
& +10 \mu^{3} z^{3}-50 \mu^{3} z+4 \mu^{2}+21 \mu^{2} z^{2}-24 \mu z+9 .
\end{aligned}
$$

Hence $C=0$ implies $\alpha^{2} r^{2}-\beta^{2}=0$, which is a polynomial expression in $(\mu, z)$ of the form $0=16\left(1-\mu^{2}\right)(1-\mu z)^{2} p_{1} p_{2}$ where

$$
\begin{aligned}
& p_{1}:=4 \mu^{2} z^{2}+5-11 \mu z+3 \mu^{2}-\mu^{3} z \\
& p_{2}:=4 \mu^{4} z^{4}-3 \mu^{5} z^{3}-\mu^{3} z^{3}+3 \mu^{4} z^{2}+\mu^{2} z^{2}-2 \mu^{5} z-9 \mu^{3} z-\mu z+6 \mu^{4}+\mu^{2}+1 .
\end{aligned}
$$

If we define $y:=\mu z$, we have $y \in[0,1)$, and

$$
p_{2}=(6-2 y) \mu^{4}+\left(-3 y^{3}+1+3 y^{2}-9 y\right) \mu^{2}+4 y^{4}-y^{3}-y+y^{2}+1 \text {. }
$$

The discriminant of this polynomial with respect to $\mu^{2}$ is $(9 y+23)(y+1)^{2}(y-1)^{3}<0$, so $p_{2} \neq 0$.

Similarly we have $p_{1}=(3-y) \mu^{2}+4 y^{2}+5-11 y$, hence $p_{1}=0$ implies $\mu^{2}=$ $\left(4 y^{2}+5-11 y\right) /(y-3)$. Then we get $\mu^{2}-y^{2}=(5-y)(y-1)^{2} /(y-3)<0$ which is a contradiction since $y^{2}=\mu^{2} z^{2} \leq \mu^{2}$.

This ends the proof of the lemma.

\section{References}

[1] Barbeau, E. J.: Polynomials, Springer-Verlag, 1989

[2] Belloni, M., and Kawohl, B.: A Paper of Legendre Revisited, Forum Math. 9 (1997), 655-667

[3] Brock, F., Ferone, V., and Kawohl, B.: A Symmetry Problem in the Calculus of Variations, Calc. Var. Partial Differential Equations 4 (1996), 593-599

[4] Buttazzo, G., Ferone, V., and Kawohl, B.: Minimum Problems over Sets of Concave Functions and Related Questions, Math. Nachrichten 173 (1993), 71-89

[5] Comte, M., and Lachand - Robert, T.: Existence of Minimizers for Newton's Problem of the Body of Minimal Resistance under a Single Impact Assumption, to appear in J. Anal. Math.

[6] Comte, M., and Lachand - Robert, T.: Newton's Problem of the Body of Minimal Resistance under a Single-Impact Assumption, to appear in Calc. Var. in PDE.

[7] Guasoni, P.: Problemi di Ottimizzazione di Forma su Classi di Insiemi Convessi, Tesi di Laurea, Pisa, 1996

[8] Newton, I.: Philosophiae Naturalis Principia Mathematica, 1686 
[9] Lachand - Robert, T., and Peletier, M. A.: An Example of Non-Convex Minimization and an Application to Newton's Problem of the Body of Least Resistance, to appear

Université Pierre et Marie Curie Laboratoire d'Analyse Numérique Tour $55-65,5^{\mathrm{e}}$ étage

4, place Jussieu

75252 Paris Cedex 05

France

E-mail:

lachand@ann.jussieu.fr
Centrum voor Wiskunde en Informatica

P. O. Box 94079

1090 GB Amsterdam

The Netherlands 